\title{
LES ADVERBIAUX EN POSITION PREVERBALE : PORTEE CADRATIVE ET ORGANISATION DES DISCOURS
}

\author{
Michel Charolles \\ Université de Paris III \\ UMR LATTICE ENS Ulm
}

\author{
Denis Vigier \\ Université de Rennes 2 (Cirefe) \\ UMR LATTICE ENS Ulm
}

\begin{abstract}
This paper focuses on the scope of adverbial constituents placed in preverbal zone. First, we show how writers can take advantage of the framing potential of these adverbials to structure their discourse. Then, we review the ways in which the notion of 'scope' is used in semantics. In the last section, we argue for the notion of 'framing scope' as a way to account for the organizational function these adverbials assume beyond the sentence in which they occur. A specific argument for this distinction between 'semantic scope' and 'framing scope' is that these adverbials have the capacity to include in their 'framing scope' connectors or incidental clauses from whose contents do not fall under their 'semantic scope'.
\end{abstract}

\section{RESUME}

Cet article traite de la portée des adverbiaux détachés en position préverbale. Nous montrons d'abord quels avantages les rédacteurs peuvent retirer du potentiel cadratif de ces adverbiaux pour structurer leur discours. Nous rappelons ensuite ce que l'on entend par "portée » en sémantique. Dans notre dernière partie, nous proposons d'utiliser la notion de "portée cadrative » pour rendre compte de la fonction organisationnelle des adverbiaux audelà de leur phrase d'accueil. Cette distinction entre "portée sémantique " et "portée cadrative » est d'autant plus nécessaire que les adverbiaux antéposés peuvent inclure dans leur "portée cadrative» des connecteurs et des incises dont le contenu ne tombe pas sous leur «portée sémantique ».

\section{Introduction}

Certains adverbiaux en position préverbale peuvent étendre leur influence au-delà de leur phrase d'accueil. Ils regroupent au sein de blocs (ou cadres) des informations qui satisfont au critère spécifié par l'adverbial (que ce critère soit relatif au contenu propositionnel, à l'énoncé ou à l'énonciation) et participent, de ce fait, à la cohésion du discours (M.Charolles à par.).

Quels mécanismes sont à la source de ce pouvoir ? A quelle étape de l'élaboration de la signification les relations cadratives induites par les adverbiaux antéposés sont-elles calculées ? L'influence d'un adverbial sur une ou plusieurs phrases faisant suite à celle dans laquelle il apparaît, relève-t-elle de la portée au sens où on entend habituellement cette notion en linguistique ? Les cadres regroupant plusieurs phrases indexées par un même adverbial 
introducteur sont-ils réellement "homogènes" ? C'est à ces interrogations que l'étude qui suit se propose de répondre.

Dans la première partie, nous montrons, à partir de quelques extraits tirés de l'écrit, quels avantages les rédacteurs peuvent retirer pour l'organisation de leur exposé du potentiel cadratif des adverbiaux spatiaux, temporels et praxéologiques qui se prêtent particulièrement bien à ce genre d'exploitation. Dans la seconde partie, nous rappelons comment l'idée de portée est utilisée en sémantique et nous préconisons de parler de portée cadrative pour rendre compte de la fonction organisationnelle des adverbiaux au-delà de leur phrase d'accueil. Cette distinction est d'autant plus nécessaire que, comme nous le soulignons dans la troisième partie, les adverbiaux antéposés peuvent inclure dans leur portée cadrative des connecteurs et des incises dont le contenu ne tombe pas sous leur portée sémantique.

\section{LES ADVERBIAUX EN POSITION PREVERBALE}

Dans l'extrait suivant, les SP adverbiaux (compléments circonstanciels, non essentiels, non argumentaux) antéposés pourraient être déplacés en fin de phrase sans modification apparente de la signification :

(1) "La vie partagée qu'il menait avait longtemps paru à Edouard un chef-d'œuvre d'organisation heureuse. Aux Pierres Sonnantes il se donnait tout entier aux exigences de l'usine et aux soins de Maria-Barbara et des enfants. À Paris, il redevenait le célibataire oisif et argenté de sa jeunesse. (M. Tournier Les météores)

Pour se rendre compte de l'effet et de l'intérêt de la présentation adoptée par l'auteur, il suffit cependant de rajouter à la fin du passage (1a) une suite (inventée) et de comparer avec la version où les SP seraient postposés $(1 \mathrm{~b})$ :

(1a) La vie partagée qu'il menait avait longtemps paru à Edouard un chef-d'œuvre d'organisation heureuse. Aux Pierres Sonnantes il se donnait tout entier aux exigences de l'usine et aux soins de Maria-Barbara et des enfants. À Paris, il redevenait le célibataire oisif et argenté de sa jeunesse. Il se couchait très tard et dormait peu.

(1b) La vie partagée qu'il menait avait longtemps paru à Edouard un chef-d'œuvre d'organisation heureuse. Il se donnait tout entier aux exigences de l'usine et aux soins de Maria-Barbara et des enfants aux Pierres Sonnantes. Il redevenait le célibataire oisif et argenté de sa jeunesse à Paris. Il se couchait très tard et dormait peu.

La version (1a) invite à penser que c'est seulement quand le personnage est à Paris qu'il se couche tard et dort peu, alors que, dans la version (1b), on ne peut pas du tout décider si le segment rajouté fait allusion au mode de vie qu'il adopte à Paris ou à celui qui est le sien en général (i.e. dans la capitale aussi bien qu'aux Pierres Sonnantes).

Le fait que la version (1a) soit comprise ainsi qu'on vient de le voir montre que les adverbiaux détachés en tête de phrase sont à même d'étendre leur influence à une ou plusieurs phrases faisant suite à celle dans laquelle ils figurent. Cette prérogative est tout à fait remarquable dans la mesure où elle ne joue pas avec les SP postposés qui ne sont pas en mesure d'exercer une influence comparable en amont ${ }^{1}$. Cela apparaît clairement dans (1c) où l'allusion aux habitudes du personnage en matière de sommeil ne peut pas être comprise comme valant seulement pour son comportement à Paris puisque ce lieu n'a pas encore été évoqué :

(1c) La vie partagée qu'il menait avait longtemps paru à Edouard un chef-d'œuvre d'organisation heureuse. Il se donnait tout entier aux exigences de l'usine et aux soins

\footnotetext{
${ }^{1}$ Cf. Cette différence est bien vue notamment par Thompson (1985). Pour une présentation discussion de Thompson et des travaux d'inspiration fonctionnaliste proches, cf. Charolles \& Lamiroy (2002), Charolles (2003).
} 
de Maria-Barbara et des enfants aux Pierres Sonnantes. Il se couchait très tard et dormait peu. Il redevenait le célibataire oisif et argenté de sa jeunesse à Paris.

Quand on compare (1c) à (1d) :

(1d) La vie partagée qu'il menait avait longtemps paru à Edouard un chef-d'œuvre d'organisation heureuse. Aux Pierres Sonnantes, il se donnait tout entier aux exigences de l'usine et aux soins de Maria-Barbara et des enfants. Il se couchait très

tard et dormait peu. À Paris, il redevenait le célibataire oisif et argenté de sa jeunesse. on voit immédiatement la différence. Autant (1c) paraît mal organisé, autant (1d) coule de source.

Cette impression s'explique par plusieurs raisons. Dans la version (1c), le SP (aux Pierres Sonnantes) en position finale apporte une information complémentaire sur le lieu où s'exerce le comportement du personnage. La phrase soulignée prolongeant la description de ce comportement, l'indication spatiale passe au second plan et tend à être reconduite par le lecteur. Rien ne permettant à ce dernier de considérer que le nouveau comportement mentionné (il redevenait le célibataire oisif et argenté de sa jeunesse) prendrait place dans un autre endroit, il tend à considérer qu'il vaut également pour les Pierres Sonnantes, inférence qu'il doit corriger lorsqu'il prend connaissance, en fin de phrase, du SP à Paris. La version (1d) qui évite une telle réanalyse est d'emblée plus coopérative. Elle présente en outre l'avantage supplémentaire que, comme dans la version originale, l'allusion aux lieux apparait plus motivée que dans la version (1c) où, jusqu'à l'occurrence du second SP, on ne voit pas clairement à quelle intention répond l'allusion au fait que c'est aux Pierres Sonnantes qu'il s'adonne aux occupations mentionnées. Dans (1d), l'occurrence du second SP antéposé est comprise comme signalant que les états de choses évoqués dans les deux phrases précédentes ne valaient que pour ce qui se passe aux Pierres Sonnantes et la phrase rajoutée est comprise sans la moindre hésitation comme tombant sous le coup du SP aux Pierres Sonnantes. Avec (1a), quoique la mise en place successive des deux SP incite, comme signalé un peu plus haut, à comprendre que c'est à Paris seulement que le personnage se couche tard et dort peu, on ne peut toutefois pas complètement exclure que la phrase rajoutée vaille pour son comportement en général. Nous reviendrons sur ce point dans la dernière partie.

$\grave{A}$ propos de (1) et de (1d) on notera encore que, dans ces deux versions, les SP prennent une valeur temporelle et conditionnelle (quand il était aux Pierres Sonnantes, il ... Quand il était à Paris, il ....), alors que, quand ils sont postposés et en position rhématique, ainsi que dans (1c), ils gardent leur valeur spatiale ${ }^{2}$. Ce déplacement de sens et l'effet de contraste qui va de pair renvoie à l'allusion, dans la première phrase de l'extrait reproduit, au fait que le personnage a une "vie partagée". Cette première phrase n'est pas indispensable à l'interprétation de (1) : on pourrait la supprimer et on pourrait même imaginer que le roman commence par : Aux Pierres Sonnantes Edouard se donnait tout entier aux exigences de l'usine et aux soins de Maria-Barbara et des enfants. À Paris, il ... . Il n'empêche qu'elle facilite la lecture en ce qu'elle justifie le détachement en position de topique des deux SP, détachement qui offre ensuite la possibilité de répartir les informations sous deux rubriques (Prévost 2003, Charolles 2003).

Dans (1), la capacité des SP détachés en tête de phrase à indexer plusieurs phrases est sous-exploitée de sorte que le schéma de composition textuelle que l'on vient de dégager n'a qu'une portée restreinte. Dans l'extrait suivant, tiré d'un corpus très différent, on retrouve une construction comparable avec une phrase amorce à l'initiale de paragraphe précédant les deux SP faisant allusion à des domaines d'activités :

(2) Les sucres viennent aussi influencer nos perceptions de la texture des aliments, soit la «sensation en bouche». Par exemple, les sirops de glucose dans la

\footnotetext{
${ }^{2}$ Huumo (1996 et 1999).
} 
crème glacée donnent du corps et de la texture, ce que l'on perçoit comme une douceur. Ces sirops aident à prévenir la cristallisation du lactose, qui donnerait la texture sableuse ou granuleuse qu'on retrouve parfois dans certains produits laitiers surgelés.

En confiserie, le contrôle de la vitesse et de la durée de la cristallisation du sucre permet de varier les textures. En minimisant la cristallisation on obtient les textures douces des fondants et des fudges. Les bonbons durs résultent d'une cristallisation qui donne une structure granuleuse ou cristalline. Le miel, grâce à ses propriétés de non-cristallisation, s'utilise pour obtenir une consistance molle et lisse.

En boulangerie, à mesure que l'on enlève l'eau pendant la cuisson, le sucre se cristallise à nouveau pour donner une texture craquante. Cette texture se voit renforcée par le brunissement (réaction de Maillard) qui se produit lorsque l'on chauffe ensemble des sucres réducteurs ( $p$. ex. le glucose et le fructose) et des ingrédients contenant de l'azote (p. ex. les protéines).

Les sucres servent aussi à attendrir les produits de boulangerie en ralentissant le rythme auquel les molécules d'amidon se lient et celui auquel les protéines se dégradent....(Web)

Dans (2), les SP antéposés indexent l'ensemble des informations exprimées par les phrases composant les paragraphes en tête desquels ils sont détachés. Ils initient des cadres dans lesquels sont réparties les informations, ce que l'on peut schématiser comme suit :

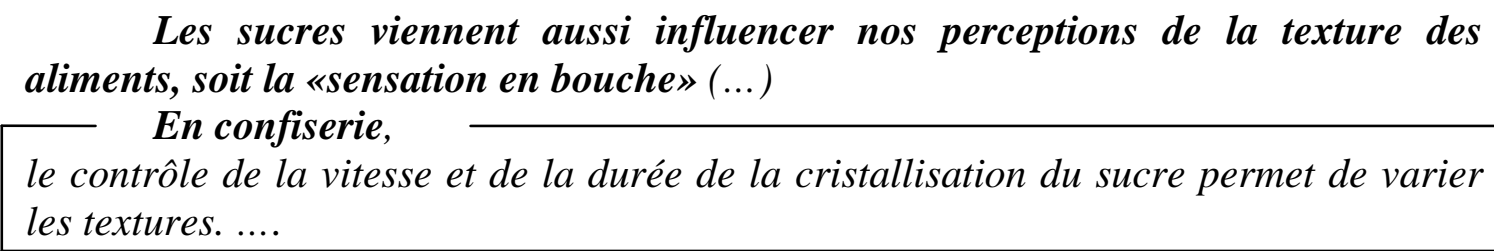

En boulangerie,

à mesure que l'on enlève l'eau pendant la cuisson, le sucre se cristallise à nouveau pour donner une texture craquante....

Les sucres servent aussi à attendrir les produits de boulangerie ...

Les SP fixent un critère en fonction duquel les données dont il est fait état dans le texte sont classées. Ce critère n'est pas strictement spatial, il fait référence à un secteur ou domaine d'activité. Les cadres "praxéologiques" (Vigier 2003, 2004) initiés par les deux SP regroupent des informations illustrant la façon dont les sirops de glucose permettent de contrôler la cristallisation des produits dans la fabrication desquels ils entrent, sujet qui constitue, à ce point du texte, le topique de discours local. Les SP ne participent pas ou, tout du moins, pas directement (Charolles 2003, Prévost 2003), à la mise en place de ce topique qui est annoncé dans la phrase amorce. Quoique non parenthétiques, ils acquièrent, du fait de leur position incidente en zone préverbale (cf. Bonami et alii à par.), une fonction organisatrice originale : le rédacteur sélectionne un élément du contenu propositionnel des énoncés pour distribuer ceux-ci dans des blocs informationnels homogènes.

La mise en place d'une telle organisation implique que le rédacteur anticipe le bénéfice qu'il peut retirer pour la clarté de son exposé, du fait que les informations touchant au sujet dont il traite présentent certaines caractéristiques communes. Cette démarche, éminemment stratégique, est difficilement compatible avec les conditions de production que l'on rencontre 
dans les conversations impromptues. A l'écrit, où le locuteur a "tout loisir" de planifier et réviser son propos, les mises en forme de ce type sont plus aisées, avec parfois des choix qui sont intéressants à observer ${ }^{3}$. Il en va ainsi, nous semble-t-il, dans l'extrait suivant :

(3) "L'un des éléments qui a permis à l'archéologie de se développer est de faire siennes les méthodes d'analyses scientifiques. L'intérêt des archéologues s'est détourné de la forme des objets échangés pour se porter sur le matériau qui les constituait. Grâce à la chimie et à la pétrographie, on peut maintenant identifier de manière très précise toutes les roches, et souvent déterminer exactement leur lieu d'origine. Il est donc aujourd'hui possible d'établir, d'où vient la pierre qui a servi à fabriquer la hache ou le vase trouvé sur un site donné. Autrefois, les archéologues comparaient des formes : lorsqu'ils déterraient un objet d'aspect inhabituel, ils cherchaient sur d'autres sites des objets de forme identique, ou des «parallèles ». Quand ils en avaient trouvé, ils expliquaient ces similitudes par l'existence de relations commerciales. Dans certains cas, ces similitudes étaient purement fortuites. En effet, des artisans travaillant en des lieux différents avaient très bien pu, par pur hasard, créer une même forme ou faire une même découverte, ce qui égarait les déductions. Il n'y avait absolument aucun moyen de déterminer si ces "parallèles » étaient pertinents ou non."

Dans (3), le SP indexe les deux infinitives compléments de pouvoir et la phrase qui suit. Le critère retenu fait état des moyens que les chercheurs sont en mesure de mettre en œuvre, mais, en même temps le texte fait allusion aux circonstances temporelles de cette mise en œuvre à l'aide de maintenant. Cet adverbe n'étant pas en position cadrative, le rédacteur est contraint de le reprendre dans la phrase suivante au moyen de aujourd'hui qui n'est pas non plus en position cadrative, au contraire du autrefois qui suit. Partant, on voit bien comment l'auteur aurait pu profiter de cette opportunité pour organiser son exposé de façon plus économique et sans doute plus efficace, en exploitant conjointement le potentiel cadratif et contrastif des adverbes temporels ainsi que dans :

(3a) L'un des éléments qui a permis à l'archéologie de se développer est de faire siennes les méthodes d'analyses scientifiques. L'intérêt des archéologues s'est détourné de la forme des objets échangés pour se porter sur le matériau qui les constituait.

Maintenant, grâce à la chimie et à la pétrographie, on peut identifier de manière très précise toutes les roches, et souvent déterminer exactement leur lieu d'origine. Il est donc aujourd'hui possible d'établir, d'où vient la pierre qui a servi à fabriquer la hache ou le vase trouvé sur un site donné. Autrefois, les archéologues comparaient des formes (...)

Une des raisons expliquant que le rédacteur n'ait pas profité de cette opportunité tient probablement au fait que les deux phrases au début de l'extrait mettent en avant d'abord les "méthodes scientifiques" et seulement ensuite l'évolution dans le temps des "intérêts des archéologues". Cette amorce favorise le détachement en premier lieu du SP de moyen ainsi que dans la version originale. Mais, une fois ce choix effectué, le détachement en position cadrative des adverbiaux temporels devenait difficile, si ce n'est impossible, pour la simple raison qu'une disposition comme :

(3b) L'un des éléments qui a permis à l'archéologie de se développer est de faire siennes les méthodes d'analyses scientifiques. L'intérêt des archéologues s'est détourné de la forme des objets échangés pour se porter sur le matériau qui les constituait.

\footnotetext{
${ }^{3}$ Ces mises en forme ne sont cependant pas absentes de l'oral. Les segments détachés en tête de séquences ("périodes", "paragraphes") d'énoncés, dits "préfixes" par Blanche-Benveniste, Bilger \& Van der Eynde (1990), "préambules" par Danon-Boileau \& Morel (1994), et "têtes" par Lacheret, Floux \& Victorri (1998), Lacheret \& Victorri (2002) peuvent parfaitement comporter des adverbiaux ("cadres").
} 

organisation des discours », Langue Française, 148, 9-30.

Grâce à la chimie et à la pétrographie, maintenant, on peut (...). Autrefois, les archéologues comparaient (...).

aurait inévitablement amené à subordonner le second cadre temporel introduit par autrefois sous le cadre initié par grâce à (...) et donc à comprendre, de façon contradictoire, que les archéologues utilisaient déjà dans le passé des méthodes empruntées à la chimie et à la pétrographie.

Les observations présentées dans cette partie montrent que les adverbiaux en position préverbale jouissent d'un potentiel cadratif qui leur est propre et que ce potentiel est souvent utilisé par les locuteurs pour répartir les informations communiquées dans des rubriques qui sont typées par l'adverbial. On a vu également que les cumulations d'adverbiaux de types différents ne sont pas rares avec des effets de placement qui sont contraignants. Ces contraintes résultent à la fois de la façon dont l'antéposition des adverbiaux est amorcée par le contexte amont et des effets de la subordination d'un cadre sous un autre. Lorsqu'en effet on considère la façon dont les cadres peuvent se mettre en place au fur et à mesure de l'avancée du discours (Charolles 1997) on constate :

- qu'ils ont tendance, par défaut, à intégrer les informations arrivantes, leur fermeture étant conditionnée par l'occurrence d'un ou, le plus souvent, de plusieurs indice(s) cotextuel(s) à même de signaler que le cadre actif n'a plus cours (cf. néanmoins Le Draoulec \& Péry-Woodley, ce volume),

- que cet indice est fréquemment un introducteur du même type (cf. la succession des SP spatiaux dans (1), praxéologiques dans (2) et temporels dans (3a)) mais que cela est loin d'être toujours le cas (cf. (2) où le cadre initié par en boulangerie n'est pas fermé par un nouvel introducteur praxéologique mais par l'alinéa, et par un changement $\mathrm{du}$ topique de discours),

- que la cumulation de cadres de types différents peut conduire à des effets de subordination en fonction de leur ordre d'apparition (cf. (3b) où les cadres temporels sont subordonnés au cadre introduit par grâce à ...).

\section{PORTEE SEMANTIQUE ET PORTEE CADRATIVE}

Pour rendre compte de l'influence que peut exercer un adverbial antéposé au-delà de sa phrase d'accueil, on est tenté de recourir à la notion de portée. Mais ce terme convientil vraiment?

Rappelons tout d'abord qu'en logique, la portée (ou "scope") d'un opérateur désigne le fragment d'une formule donnée auquel il s'applique. Par convention, les parenthèses permettent (quand cela est nécessaire) de délimiter la portée, ainsi que dans :

$$
\urcorner\left(\mathrm{p}^{\wedge} \mathrm{q}\right)
$$

où la négation (\urcorner$)$ s'applique, dans le premier cas, à la sous formule $\left(\mathrm{p}^{\wedge} \mathrm{q}\right)$ et dans le second uniquement à $\mathrm{p}^{4}$.

A l'inverse des langages formels, les langues naturelles ne possèdent pas de dispositifs conventionnels destinés à expliciter de manière univoque la portée des unités à portée (désormais UP). En linguistique, la portée d'une UP est en général définie comme le (ou les) constituants $^{5}$ de l'énoncé sur lequel (lesquels) cette unité exerce une influence sémantique ${ }^{6}$.

\footnotetext{
${ }^{4}$ Cf. entre autres Rivenc (1989).

${ }^{5}$ Muller $(1991: 101 ; 2005: 242)$ considère que la portée d'un opérateur affecte un «domaine » et non un constituant.

${ }^{6} \mathrm{~T}$. Huumo par exemple définit le scope d'un locatif comme « its semantic coverage of other elements in the sentence. » (1996: 266)
} 
Relèvent classiquement d'une analyse en termes de portée la quantification, les verbes d'attitude propositionnelle et la négation :

(4) Deux enfants ont mangé trois gâteaux (Danell, 1998 : 5)

(5) Marie se figure que Paul a démissionné parce que les journaux parlent de lui.

(6) Il ne pique-nique pas dans la forêt

Deux interprétations de (4) sont possibles suivant que l'on considère que le quantificateur trois tombe sous la portée du quantificateur deux (deux enfants, six gâteaux) ou non (deux enfants, trois gâteaux), ce qui donne deux lectures dites respectivement distributive et collective. Dans (5), le verbe d'attitude propositionnelle se figure peut porter soit sur l'événement dénoté par Paul a démissionné (glose : Les journaux parlent de Paul ; voilà pourquoi Marie se figure qu'il a démissionné ), soit seulement sur la relation causale (glose : Marie se figure que la cause de la démission de Paul est le fait que les journaux parlent de lui, les deux énoncés présupposés n'en formant qu'un seul). Concernant (6) enfin, Neveu (2000 : 87) considérerait que la négation peut porter aussi bien sur le prédicat entier piqueniquer dans la forêt ( $a$ : Il ne pique-nique pas dans la forêt, il se repose dans la salle de séjour) que sur une partie seulement de ce dernier : le verbe (b:Il ne pique-nique pas dans la forêt, il s'y promène) ou l'adverbial locatif ( $c$ : Il ne pique-nique pas dans la forêt, mais dans le jardin). Riegel et alii. (1999: 411-412) proposeraient une analyse sensiblement proche. À l'inverse, si l'on suit Nølke (1993, 1994, 2001), la portée de la négation ne varierait pas dans (6) et s'étendrait systématiquement sur la proposition entière. Les variations d'interprétation $(a, b, c)$ explicitées ci-dessus s'expliqueraient par l'association de la négation (qui ne marque pas par elle-même un domaine de focalisation) au foyer sélectionné contextuellement à l'intérieur du domaine de focalisation que constitue pique-nique dans la forêt ${ }^{7}$. Signalons pour terminer que, si l'on suit Muller (1991), dans (6), la portée de la négation engloberait aussi l'ensemble de la proposition, ce que traduit la notation suivante ${ }^{8}$ :

Pas (dans la forêt (pique-nique (il)))

dans laquelle "tout ce qui est inclus dans les parenthèses que domine pas constitue la portée de la négation" (1991 : 105).

Outre le cas des quantificateurs, des verbes d'attitude propositionnelle et de la négation, divers auteurs comptent parmi les UP certains adverbes et adverbiaux. Guimier notamment définit la portée des adverbes en -ment comme "l'élément à propos duquel l'adverbe dit préférentiellement quelque chose" (1996 : 4). Il distingue (ibid., 5-7) les adverbes endophrastiques qui "participent à la construction du sens référentiel de la phrase" des exophrastiques qui ont pour "support de portée" les différentes étapes de l'acte d'énonciation (le dire, le dit ou la visée de discours). Par exemple, dans les trois phrases suivantes empruntées à l'auteur (ibid., 4), les adverbes en -ment endophrastiques, tous incidents syntaxiquement au verbe, ont pour portée successivement le verbe (7), le sujet (8) et le sujet + le verbe $(9)$ :

(7) Pierre travaille manuellement

(8) Pierre travaille jovialement

(9) Pierre travaille minutieusement

Ces trois portées peuvent être mises en lumière au moyen des paraphrases suivantes ${ }^{9}$ : "[Portée sur le sujet] Pierre est ( ${ }^{*}$ manuel ${ }^{10}+$ jovial + minutieux) dans son travail / [Portée sur le verbe] Le travail de Pierre est (manuel + *jovial + minutieux)". Nølke, quant à lui, différencie "l'étendue de portée" d'un adverbial - le segment de la phrase qui entre dans la

\footnotetext{
${ }^{7}$ Voir Nølke, 1993 : 243-250 ; $2001: 241$.

${ }^{8}$ Voir « la hiérarchie des prédicats sémantiques ordonnés » qu'utilise l'auteur dans (1991: 105 et sq.).

${ }^{9}$ Voir Guimier (1996 : 48, 51) ; Molinier \& Levrier (2000 : 117)

${ }^{10}$ Selon l'auteur, un énoncé comme «Pierre est manuel » est acceptable mais ne constitue pas une paraphrase de (7)
} 
portée du constituant à portée - et "la perspective de portée", c'est-à-dire "l'aspect sous lequel ce segment [= l'étendue de portée] est vu" (2001 : 244). Par exemple l'adverbe d'énonciation franchement placé en tête d'une proposition $p$ a pour étendue, explique-t-il, toute cette proposition et pour perspective "l'illocutoire".

La position antéposée d'un adverbial - lorsqu'elle est possible - tend à modifier ses caractéristiques syntaxiques et sa portée sémantique. Voici deux exemples :

(10a) Max répond à la question prudemment

(10b) Prudemment, Max répond à la question

Sur le plan syntaxique, (10b), à l'inverse de (10a), est incompatible avec les modalités interrogative (interrogation totale) et injonctive ${ }^{11}$. Sémantiquement, l'adverbe, qui possède une portée sur le verbe et le sujet dans (10a), voit celle-ci s'étendre sur la proposition entière dans (10b). Les exemples (11a) et (11b) sont empruntés à Huumo (1996:270) :

\section{(11a) Elmeri a vu Anselme dans le bus}

(11b) Dans le bus, Elmeri a vu Anselme

Dans (11a), le locatif postposé a pour portée soit Anselme soit la scène entière (Elmeri et Anselme sont dans le bus), mais pas, ou très difficilement, Elmeri seulement. Par contre, le locatif antéposé dans (11b) peut porter soit sur la scène entière, soit sur Elmeri seul, l'adverbial prenant, dans ce second cas, une valeur temporelle ${ }^{12}$.

Le changement de position de l'adverbial peut cependant n'exercer aucune influence sur l'interprétation des relations de portée à l'intérieur de la phrase. Par exemple, dans :

(12a) Deux examinateurs préparaient un sujet chaque matin

(12b) Chaque matin, deux examinateurs préparaient un sujet

deux interprétations sont à chaque fois possibles : (i) "il se présente chaque matin deux examinateurs qui préparent deux sujets" (lecture distributive ; le quantificateur un tombe sous la portée du quantificateur deux); (ii) "il se présente chaque matin deux examinateurs qui préparent ensemble un seul sujet" (lecture collective : pas de portée de deux sur un). Si l'on spécifie maintenant au moyen d'une phrase amont la durée de la session ( $L$ 'examen dura cinq jours. (Chaque matin,) deux examinateurs préparaient un sujet (chaque matin)), on arrive à un grand nombre d'interprétations. On peut envisager, en effet, soit que ce sont les deux mêmes examinateurs qui ont composé tous les sujets de la session (deux examinateurs), soit qu'ils étaient différents chaque jour (dix examinateurs) - toutes les solutions intermédiaires étant par ailleurs possibles (par exemple, on a changé une fois les examinateurs durant la session). De surcroît, il faut envisager pour chacune de ces solutions deux possibilités : soit un seul sujet par jour, soit deux, ce qui fait qu'on peut dénombrer au moins dix interprétations possibles pour les énoncés (12a) ou (12b). Il est évident que l'auditeur ne passe pas en revue toutes ces interprétations lorsqu'il traite un tel énoncé : les besoins de la communication s'accommodent en général d'un tel "vague", fait remarquer Muller (1991 : 111), du moins tant que le contexte n'exige pas de précisions supplémentaires ${ }^{13}$.

\footnotetext{
${ }^{11}$ Guimier, 1996 : 90 ; Molinier \& Levrier, 2000 : 111-112. « Max répond-il à la question prudemment ?» versus «*Prudemment, Max répond-il à la question? » / «Que Max réponde à la question prudemment» versus "*Prudemment, que Max réponde à la question». Dans (10a), selon Molinier \& Levrier (2000), l'adverbe «prudemment » est un adverbe de «manière orienté vers le sujet » et dans (10b), un adverbe de phrase disjonctif d'attitude orienté vers le sujet.

12 T. Huumo (1996) ne détaille pas ces interprétations mais note : «A clause-initial adverbial is interpreted as introducing the scene of the whole event, whereas a similar adverbial occurring clause-finally is interpreted as introducing the scene of some entity only" (270)

${ }^{13}$ Danell (1998 : 9) suggère quant à lui que lorsqu'un énoncé présente plusieurs quantificateurs et qu'aucun d'entre eux ne possède un sens explicitement distributif - ce qui n'est pas le cas ici, puisque nous avons « chaque »-, l'auditeur choisit par défaut (et dans un contexte neutre) l'interprétation collective. Ainsi, dans une phrase comme «35 enfants ont mangé 5 gâteaux sur 3 tables », le sujet tend à sélectionner la lecture : «35 enfants, 5 gâteaux, 3 tables », solution qui entraîne un minimum de calculs arithmétiques.
} 
La comparaison de (12a) et (12b) montre que la position de l'adverbial chaque matin au sein de la phrase n'a pas d'incidence sur le calcul des relations de portée entre les divers quantificateurs intraphrastiques. En revanche, cette position devient cruciale lorsqu'on s'avise de regarder ce qui se passe au-delà de la phrase. On s'aperçoit en effet que l'antéposition frontale de certains adverbiaux permet au trait sémantique qu'ils expriment de jouer un rôle de critère de regroupement des informations au-delà de leur phrase d'accueil, comme on l'a souligné en début d'article. On peut aisément le montrer de nouveau ici en modifiant les énoncés (12a) et (12b) en (12c) et (12d) :

(12c) La session dura cinq jours ${ }_{[p]}$. Chaque matin, deux examinateurs préparaient un sujet $_{[q] .}$. Le Directeur en prenait connaissance ${ }_{[r]}$ avant de le/les faire taper par la secrétaire $_{[s]}$. Chaque soir, l'intendant affectait les salles pour l'examen $[t]$.

(12d) La session dura cinq jours ${ }_{[p]}$. Deux examinateurs préparaient un sujet chaque matin $_{[q] .}$. Le Directeur en prenait connaissance ${ }_{[r]}$ avant de le/les faire taper par la secrétaire $_{[s]}$. Chaque soir, l'intendant affectait les salles pour l'examen ${ }_{[t]}$.

Dans (12c), le lecteur tend ${ }^{14}$ à intégrer les événements dénotés par $[r$ et $s]$ dans l'intervalle temporel dénoté par chaque matin, tandis qu'avec (12d) il est tout à fait impossible de décider à quel moment le directeur intervient (matin ou après-midi ?). Ajoutons que dans (12c) et (12d), la sélection du clitique le/les (dans s) a une incidence rétroactive sur le calcul des relations de quantification mentionnées ci-dessus.

Dans la suite de cet article, nous réserverons le terme de portée sémantique pour décrire :

- les phénomènes d'ambiguïté interprétative liés à l'usage de certaines unités à portée comme les quantificateurs, la négation, les verbes d'attitude propositionnelle, ou certains adverbiaux ;

- l'influence à distance (i.e. au-delà de sa phrase d'accueil) du trait sémantique spécifié par un adverbial introducteur de cadre.

En revanche, par portée cadrative nous entendrons l'ensemble des phrases qui appartiennent toutes à un même cadre de discours ${ }^{15}$.

\section{LA PORTEE CADRATIVE}

La capacité qu'ont certains adverbiaux antéposés d'étendre leur influence au-delà de leur phrase d'accueil étant liée à leur usage fonctionnel (i.e. à leur exploitation pour la répartition des informations textuelles au fur et à mesure du discours), on peut s'attendre à ce qu'ils incluent dans leur portée cadrative des segments qui ne relèvent pas de leur portée sémantique. C'est effectivement ce qui se passe, comme nous allons le montrer dans cette partie, à propos des connecteurs et des incises.

\subsection{Portée cadrative et connecteurs}

Dans l'exemple qui suit, le SP spatial antéposé indexe les deux phrases coordonnées par mais : on comprend que la météo annonce du mauvais temps dans le Midi de la France,

\footnotetext{
${ }^{14}$ Il ne s'agit comme précisé à propos de (1) que d'une tendance, pour une discussion, cf. Le Draoulec \& Péry Woodley (ce volume).

${ }^{15}$ Dans leur classification des adverbes en -ment, Bonami et alii. (2004) distinguent la classe des "frame adverbs" (ex : légalement, théoriquement, syntaxiquement, ...) qui "explicit the domain (...) in which a sentence is to be evaluated". Ces "frame adverbs" correspondent à la catégorie des adverbes dits de "point de vue" dans la classification de Molinier et Levrier (2000) et "praxéologiques" chez Vigier (2004 et ci-avant).

${ }^{18}$ Cf. Lahousse (2003).
} 
que cette prévision va à l'encontre de l'amélioration escomptée, et que l'ensemble oriente vers l'idée que, par exemple, la saison touristique est mal engagée :

(13) "[Dans le Midi, les incendies ont cessé $]_{P 1}$ mais [la météo annonce du mauvais temps $]_{P 2}$ " (entendu à la radio)

$\mathrm{Si}$ on considère le contenu propositionnel $(p)$ de la phrase P1 en se demandant dans quel contexte elle peut être employée, les deux situations suivantes (au moins) sont envisageables :

(i) des feux dévastent plusieurs régions de la France (présupposition), le journaliste veut informer les auditeurs que les incendies ont été stoppés dans le sud du pays mais pas ailleurs : le SP fait partie du foyer de l'information qui inclut ont cessé et dans le Midi,

(ii) des feux dévastent le Midi de la France (présupposition) et le journaliste veut signaler qu'ils ont cessé : le foyer est limité au verbe.

Dans le contexte (i), le SP dans le Midi ne fait pas partie des connaissances supposées actives au moment de l'énonciation, il est rhématique et on s'attendrait à ce qu'il figure à la suite du verbe. En revanche, dans le contexte (ii), le SP ne fait pas partie du foyer et il est antéposable. En plus de ces deux situations, on peut en imaginer une troisième :

iii) le journalise n'exploite aucune information présumée active, sauf celle consistant à considérer que les auditeurs s'attendent à ce que le bulletin d'information fasse état d'événements qui se passent dans différents endroits et en particulier dans les différentes régions du pays. Le SN défini les incendies est alors accommodé (l'auditeur infère : les incendies qui s'étaient déclarés).

Dans les contextes (ii) \& (iii), l'antéposition du SP est possible, si ce n'est attendue, et P1 répondrait à la question que s'est il passé dans le Midi ? Par contre, dans le contexte (i), le SP répondrait à la question : où les incendies ont-ils cessé ? On s'attendrait plutôt à le voir postposé, à moins qu'il ne contraste avec un autre SP comme dans :

(13a) [Dans le Midi, les incendies ont cessé $]_{P 1}$ mais, [en Bretagne, ils ont empiré $]_{P 2}$ La relation établie dans (13a) entre le contenu des SP et celui des phrases en tête desquelles ils apparaissent peut être représentée comme suit :

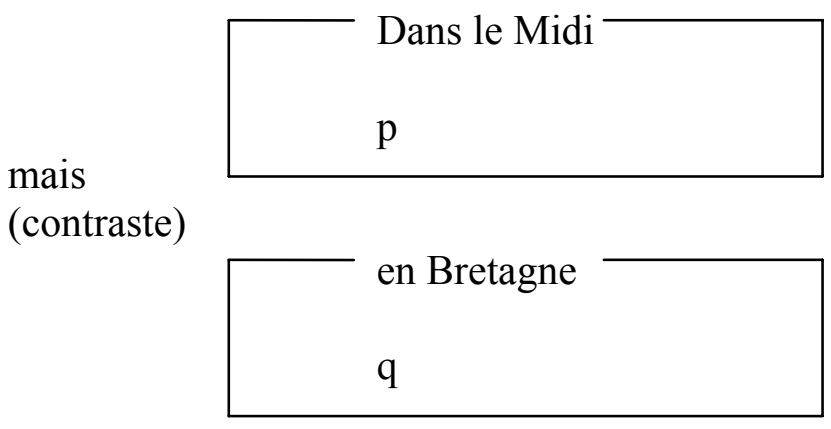

(Figure 4)

(13a) deviendrait inconsistant en cas de suppression du second SP (*Dans le Midi, les incendies ont cessé mais ils ont empiré) et il en irait de même si le second SP renvoyait à une zone de l'espace incluse dans le Midi (dans le Var), sauf à modifier l'enchaînement comme suit :

(13b) [Dans le Midi, les incendies ont cessé] ${ }_{P 1}$ mais, [dans le Var, la météo annonce du mauvais temps $]_{P 2}$

L'énoncé (13b), dans lequel mais signale une relation concessive, peut alors être schématisé ainsi : 


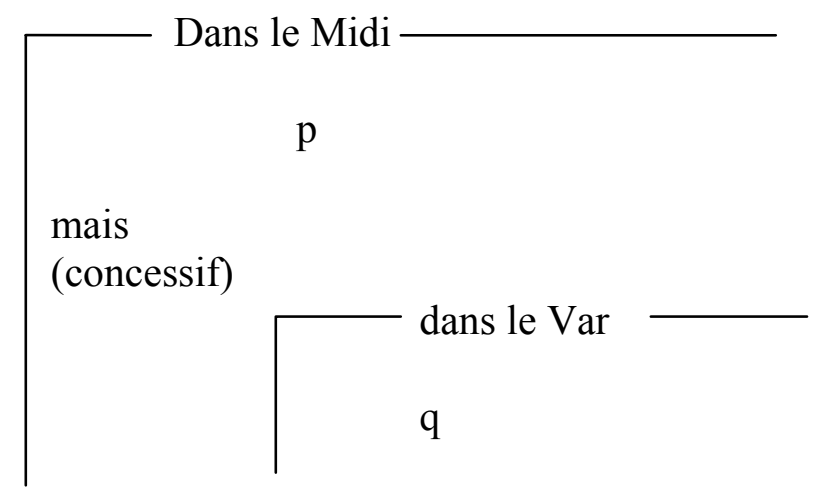

(Figure 5)

L'énoncé de départ (13) se représente de la même manière avec un mais concessif :

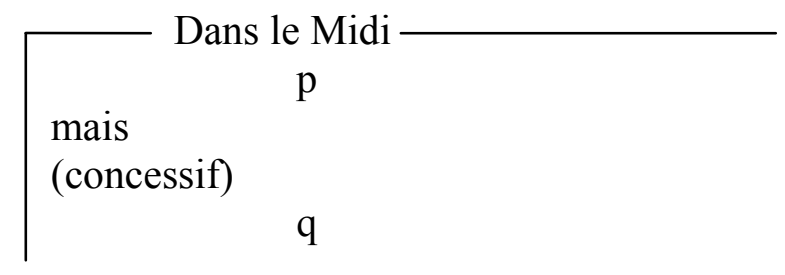

(Figure 6)

Dans les figures 4,5 et $6, p$ et $q$ notent le contenu propositionnel des phrases P1 et P2 qui inclut les SP, la réplication des SP en tant qu'introducteurs de cadre ne faisant que marquer leur rôle organisateur.

Le contexte (iii) est plus ouvert que les contextes (i) et (ii) dans le sens où le SP est utilisé comme une pure rubrique d'arrière-plan. C'est aussi ce qui se passe dans le contexte (i) avec continuation contrastive, sauf que, dans ce cas, le contenu de la seconde proposition doit développer le topique incendie, alors que dans le contexte (iii), l'enchaînement est plus libre puisqu'on pourrait avoir :

(13c) Dans le Midi, les incendies ont cessé, mais le maire de Marseille a démissionné. avec un mais concessif, mais plus difficile à interpréter qu'en (13). En l'absence de connecteur, tous les enchaînements seraient possibles. Le fait cependant que la relation (de contraste ou de concession) indiquée par mais ne puisse pas facilement impliquer les lieux seuls, comme on le voit bien dans :

(13d) ?? Dans le Midi, les incendies ont cessé mais, en Bretagne, le maire de Brest a démissionné.

montre que les connecteurs mettent toujours en cause les contenus propositionnels.

Est-il légitime d'inscrire dans les figures 5 et 6 le connecteur sous la portée du cadre initié par le SP locatif? La question ne manque pas de se poser car tout ce qui est avéré dans le Midi c'est que les incendies ont cessé et que la météo annonce du mauvais temps, mais pas qu'il y a une opposition entre ces deux faits. Le mais est rajouté aux états de choses et ce rajout ne devrait pas pouvoir être localisé quelque part. Il renvoie au locuteur qui met en relation les événements, mais il ne localise pas l'opération de pensée signalée par son emploi. Quand on se place au niveau informationnel où il s'agit de classer des informations - ce à quoi, de notre point de vue, servent fondamentalement les SP cadratifs - il en va tout autrement, car rien n'empêche de ranger dans le fichier étiqueté par dans le Midi toutes les informations ayant rapport à cette région. 
Les mêmes phénomènes peuvent être observés dans des séquences préfixées par des introducteurs de cadres de types différents. Dans :

(14) "A la Hague, l'eau de mer est contaminée, selon Greenpeace" (Le Monde, 21/06/97)

comme le SP médiatif (Schrepfer-André, 2004) est postposé, il y a affectation après coup de l'énoncé à l'énonciateur spécifié par le SN régime du selon. L'adjonction à (14) d'une suite comme :

(14a) A la Hague, l'eau de mer est contaminée, selon Greenpeace. Elle est dangereuse à la consommation.

amène à comprendre que l'assertion rajoutée $q$ n'est pas imputable à Greenpeace mais au locuteur $\left(\mathrm{L}^{\circ}\right)$ ou, plus précisément, à un énonciateur $\left(\mathrm{EL}^{\circ}\right)$ porte-parole de la vérité (cf. Charolles 1997, Charolles \& Pachoud 2001), ce que l'on peut représenter comme suit :

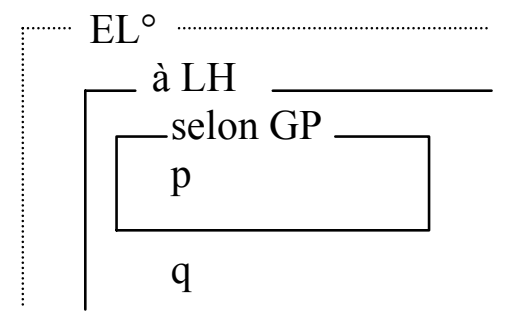

(figure 4)

Si l'on remonte le SP médiatif en tête de phrase ou à la suite du SP spatial, l'inscription de $q$ dans le cadre énonciatif initié par selon Greenpeace ne pose aucun problème et même s'impose dans les deux cas :

(14b) Selon Greenpeace, à la Hague, l'eau de mer est contaminée. Elle est dangereuse à la consommation.

(14c) A la Hague, selon Greenpeace,l'eau de mer est contaminée. Elle est dangereuse à la consommation.

Ce que l'on peut représenter ainsi :

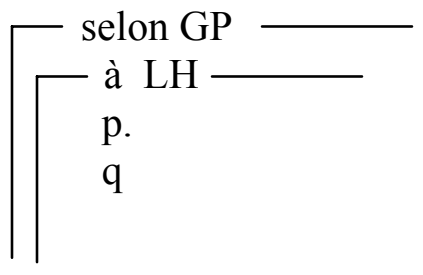

(Figure 5)

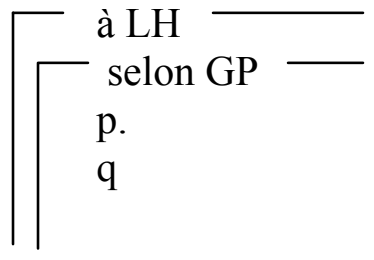

(Figure 6)

Bien que la disposition des cadres ne soit pas la même dans les deux emplois, l'emplacement des SP n'a pas d'effet sur les possibilités d'intégration de $p$ et $q$. Dans (14b), le SP locatif n'est pas imputé à Greenpeace et dans (14c) on n'infère pas que Greenpeace aurait déclaré $p$ et $q$ à la Hague. Le fait que le principe d'iconicité de placement ne fonctionne pas dans les emplois comme $(14 b)$ et $(14 c)$, contrairement à ce que nous avions noté dans la première partie à propos de (3b) où l'adverbial de moyen (grâce à $S N$ ) incluait dans sa portée les SP temporels, suggère que toutes les suites de SP cadratifs ne sont pas équivalentes pour ce qui est de leur capacité d'intégration et ne présentent pas les mêmes avantages pour l'organisation des informations. Quoi qu'il en soit de ce point qui appellerait une étude approfondie à la lumière notamment de Bonami et alii (à par.), si on rajoute à (14b) et (14d) une suite $r$ précédée d'un connecteur : mais à Caen, elle est potable, on observe une différence suivant que la 
proposition coordonnée est intégrée (intonativement /graphiquement) dans la seconde phrase (14d) ou non (14e) :

(14d) Selon Greenpeace, à la Hague, l'eau de mer est contaminée. Elle est dangereuse à la consommation, mais à Caen elle est potable.

(14e) Selon Greenpeace, à la Hague, l'eau de mer est contaminée. Elle est dangereuse à la consommation. Mais à Caen elle est potable.

Dans (14d), l'inscription de $r$ sous le cadre spatial subordonné au cadre énonciatif ne suscite aucune difficulté :

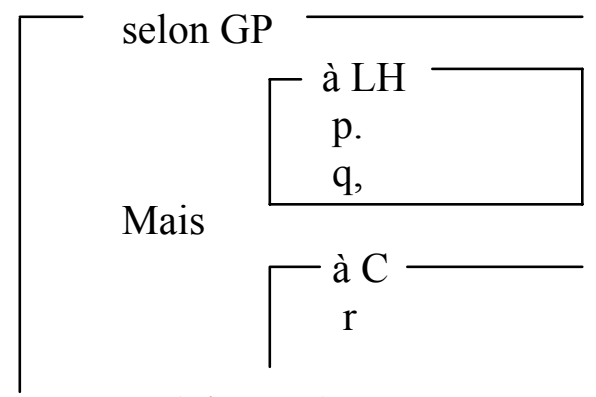

(Figure 7)

Par contre, avec (14e), il semble que l'on ait plutôt tendance à inférer que l'assertion de $r$ est le fait de $\mathrm{EL}^{\circ}$ :

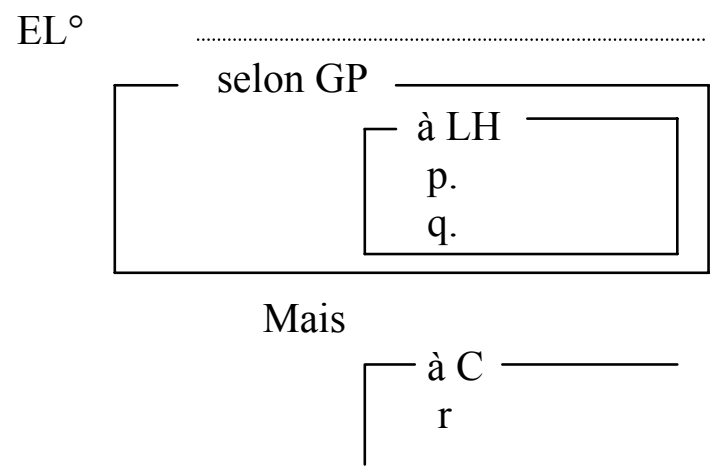

(Figure 8)

Les exemples (14d) et (14e) montrent que la ponctuation (en l'occurrence l'empaquetage des informations dans une même phrase graphique ou dans deux) joue un rôle dans le contrôle de l'extension des cadres, ce que corroborent les études sur corpus de Terran (2002) et Vigier, (2004). Dans (14d) et (14e) l'ordre de présentation des introducteurs demeure libre et on pourrait parfaitement avoir (14f) que (14g) avec une disposition des cadres schématisé respectivement sous les figures 9 et $10:$ :

(14f) A la Hague, selon Greenpeace, l'eau de mer est contaminée. Elle est dangereuse à la consommation, mais à Caen elle est potable. 


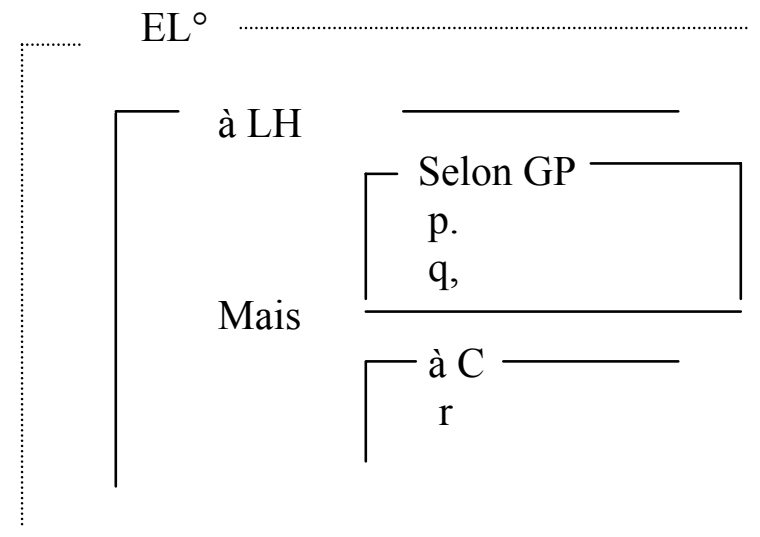

(Figure 9)

(14g) A la Hague, selon Greenpeace, l'eau de mer est contaminée. Elle est dangereuse à la consommation. Mais à Caen elle est potable.

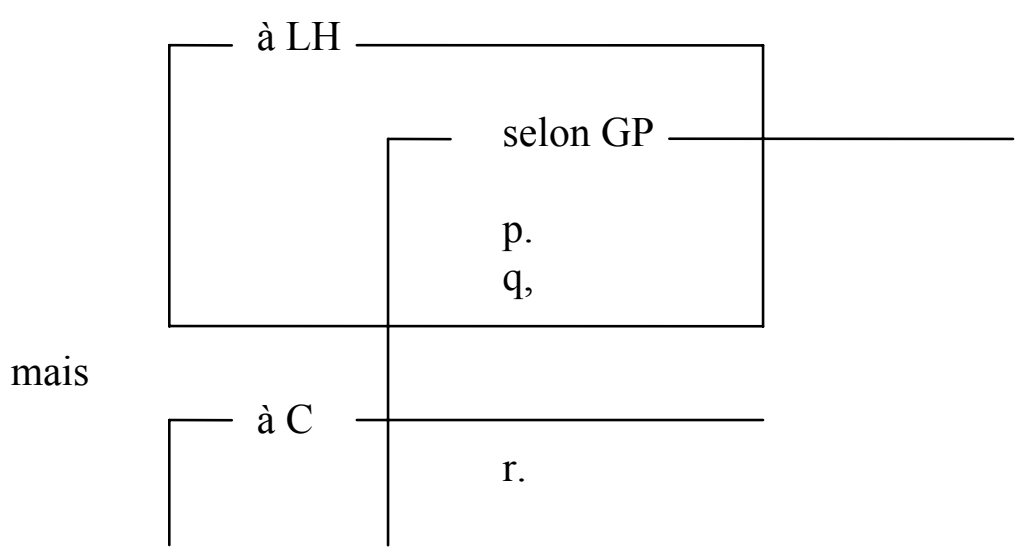

(Figure 10)

Dans (14f) le SP médiatif inclut dans sa portée cadrative $r$ aussi bien que $p$ et $q$ quoique ceuxci ne fassent pas partie du même cadre spatial. Cette disposition peut être une source potentielle de complication au niveau du traitement, de sorte qu'il n'est pas exclu que les locuteurs préféreraient (14d) plutôt que (14f)

Bien entendu, tous les connecteurs ne se prêtent pas aussi facilement que mais dans (14) à une inscription dans la portée d'un introducteur cadratif. Tout dépend du contenu sémantique à la fois de l'introducteur et du connecteur (Charolles 1997). Par exemple, dans :

(15) La Bible a une méchante réputation de violence. A en croire certains, le sang coulerait à chaque page de l'Ancien Testament. Essentiellement juge et guerrier, son Dieu ne serait pas vraiment fréquentable. A l'inverse, l'Evangile porterait un message de paix et de réconciliation universelles, davantage en accord avec l'image de Dieu révélée en Jésus.

En réalité, les choses ne sont pas si tranchées. Le premier Testament a des chapitres d'une douceur étonnante, tandis que le Nouveau ne manque pas de textes durs, même dans les évangiles. Et s'il y a bien un livre biblique dont les pages sont jonchées des victimes de la violence des hommes et surtout de Dieu, c'est l'Apocalypse de Saint Jean, le point d'orgue du Nouveau Testament. (...) (Web)

Le recours au reformulatif en réalité joint au changement de paragraphe et de temps verbal signale clairement que l'énonciateur $\mathrm{EL}^{0}$ reprend la parole pour mentionner «une 
connaissance à laquelle les autres n’ont pas accès » (voir le sens sémantico-pragmatique associé à en réalité, Rossari, 1997). En revanche, le connecteur à l'inverse tombe sans difficulté sous la portée cadrative de l'introducteur médiatif ( $A$ en croire) : il permet en effet d'opposer les opinions traditionnellement associées par la doxa au deux textes sacrés que sont l'Ancien et le Nouveau Testaments (cf. le maintien du conditionnel).

\subsection{Incises}

Dans le passage suivant, le SP en biologie initie un cadre praxéologique et, comme le propos de l'auteur est à la fois prospectif et normatif au moment où il évoque ce domaine d'étude, les segments tombant sous le coup de l'introducteur sont au futur :

(16) (...) le but que se propose la méthode expérimentale est le même partout ; il consiste à rattacher par l'expérience les phénomènes naturels à leurs conditions d'existence ou à leurs causes prochaines. [En biologie, ces conditions étant connues, le physiologiste pourra diriger la manifestation des phénomènes de la vie comme le physicien et le chimiste dirigent les phénomènes naturels, dont ils ont découvert les lois ;mais pour cela l'expérimentateur n'agira pas sur la vie.]

Seulement, il y a un déterminisme absolu dans toutes les sciences parce que (...) (C.Bernard)

Le retour au présent dans seulement, il y a un déterminisme absolu dans toutes les sciences ... signale très clairement la fermeture du cadre, fermeture qui s'accompagne (et ce n'est évidemment pas un hasard) d'un changement de paragraphe, sans compter que l'auteur revient à ce qui se passe dans les disciplines en général (dans toutes les sciences). La fixation des bornes initiale et finale du cadre (matérialisées par des crochets) ne pose donc, dans (16), aucun problème. Le fait que le cadre intègre un connecteur (mais) n'appelle pas d'autres commentaires que ceux développés dans la partie précédente. L'inscription dans la portée cadrative du SP praxéologique de la participiale (ces conditions étant connues) s'explique de la même manière dans la mesure où la construction signale une relation de justification, qui établit un lien entre la situation de la biologie au moment d'énonciation avec celle qui pourra être la sienne dans l'avenir tel que l'envisage C.Bernard. La comparative (comme le physicien et le chimiste dirigent les phénomènes naturels) et la relative qui lui est adjointe (dont ils ont découvert les lois) expriment un commentaire rajouté qui fait référence à des pratiques actuelles (cf. le présent) dans deux disciplines qui ne font pas partie de la biologie. Quoique ne tombant pas sous la portée sémantique de en biologie, l'inscription de ce commentaire dans le cadre initié par l'adverbial ne pose pas problème dans la mesure où l'information qu'il ajoute est en rapport avec ce qui se passe dans cette discipline. Ce rapport est marqué par le comparatif et par les reprises lexicales (diriger, phénomènes), de sorte que le segment rajouté, quoique en incise, est solidement accroché à la phrase en tête de laquelle le SP est détaché.

Dans l'extrait qui suit, le SP en gras introduit un cadre dont la frontière en aval peut être soit restreinte au premier crochet soit élargie jusqu'au second.

(17) (...) Ils [les chercheurs] eurent plus de succès avec une autre substance, l'EDTA (en anglais Ethylene Diamine Tetra Acetate). Cette petite molécule organique se lie si fortement aux ions calcium, qu'elle peut les arracher même s'ils sont situés dans des régions protéiques ayant elles-mêmes une forte affinité pour ces ions.[Grâce à I'EDTA, les chercheurs réussirent à solubiliser et à extraire de la paroi cellulaire un groupe de protéines insolubles quand elles y sont attachées en présence d'ions calcium - ce qui explique l'échec de la méthode précédente(1).] Les glycoprotéines* furent plus tard reconnues comme membres d'une nouvelle famille et baptisées " frustulines ", d'après le mot latin frustulum, qui désigne les coques siliceuses.] La découverte était intéressante, mais ..." (La Recherche, 2001, n³38) 
Le maintien du passé simple dans les glycérorotéines furent ... favorise la seconde solution mais cet indice n'est pas décisif étant donné que l'ensemble du passage élabore les succès des chercheurs, succès annoncés dans la phrase initiale qui est elle-même au passé simple. Quelque interprétation que l'on adopte, l'incise détachée à la suite du tiret tombe dans la portée cadrative de grâce à l'EDTA. La précision qu'elle fournit explique les succès obtenus par les chercheurs en employant l'EDTA et les échecs de ceux qui ne l'avaient pas utilisée. Cette précision adjointe se greffe directement (cf. ce qui) sur celle indexée par grâce à l'EDTA, et il en va de même avec la note (bibliographique) non reproduite. Ce rattachement s'il est "sémantiquement indu", se comprend très bien : comme il s'agit, par le biais des expressions cadratives, de répartir les informations dans des sortes de fichiers, l'adverbial qui sert d'intitulé au cadre signale simplement que les informations dans sa portée cadrative ont rapport avec l'introducteur, en l'occurrence, avec le moyen employé.

Dans le passage suivant, la restrictive soulignée tombe dans la portée du second cadre locatif :

(18) Les études menées en France font état de réductions plus faibles de l'écoulement annuel lié à la forêt : [en Bretagne du Sud, une hêtraie diminue l'écoulement de 20 pour cent par rapport aux bassins cultivés voisins.][Dans le Massif des Maures, la destruction de la végétation par des feux a entraîné, I'année suivante, une augmentation des débits annuels de presque 30 pour cent, mais cette augmentation ne s'est pas maintenue les autres années. Encore s'agit-il dans ce dernier cas de la différence entre les arbres et une maigre végétation de recolonisation, et non pas un autre couvert végétal bien établi. ]

La forêt, comparée à d'autres types de végétation, réduit ainsi le volume de l'écoulement annuel, mais de façon variable selon les conditions climatiques... (La Recherche)

Il s'agit, comme précédemment, d'une précision adjointe, mais le fait que le rédacteur ait éprouvé le besoin de préciser dans ce dernier cas est intéressant. La phrase initiale rapporte en effet une thèse qui est ensuite étayée. Les arguments invoqués ne sont pas de même nature : le premier tire parti de la baisse des écoulements des eaux en cas de peuplement forestier; le second fait état a contrario de leur augmentation quand il n'y a qu'une maigre végétation. Comme ces données soutiennent (positivement et a contrario) la thèse défendue, le rédacteur pouvait difficilement exploiter cette "opposition" pour organiser son propos, d'autant qu'il avait la possibilité de le faire, à moindre frais, en s'appuyant sur les coordonnées géographiques des états de choses. Le contenu de la restrictive annoncée par encore s'agit-il de a pour but de limiter la portée de l'argument a contrario : en effet le différentiel de $30 \%$ auquel il est fait allusion oppose un peuplement forestier à une maigre végétation de recolonisation; or on peut penser que dans le cas d'une couverture végétale bien établie, ce chiffre eût été moins important, d'où la restriction. Enfin, si le rédacteur a éprouvé le besoin de spécifier dans ce dernier cas, c'est apparemment parce que encore s'agit-il de la différence entre aurait pu être momentanément compris comme référant à la différence qu'il y a entre ce qui s'est passé en Bretagne et dans les Maures et non ce qui s'est uniquement passé dans les Maures.

Dans l'extrait suivant des Confessions de J-J Rousseau, un jour initie un cadre temporel. L'introducteur fait référence à un intervalle dont la durée est spécifiée par le SN introducteur et le cadre est fermé par le lendemain.

(19) "Un souvenir qui me fait frémir encore et rire tout à la fois, est celui d'une chasse aux pommes qui me coûta cher. Ces pommes étaient au fond d'une dépense qui, par une jalousie élevée, recevait du jour de la cuisine. Un jour que j'étais seul dans la maison, je montai sur la may pour (...). J'allai chercher la broche .... Je l'allongeai (...). Je piquai plusieurs fois sans succès; enfin je sentis avec transport que j'amenais 
une pomme. Je tirai très doucement : déjà la pomme touchait à la jalousie, j'étais prêt à la saisir. Qui dira ma douleur? La pomme était trop grosse, elle ne put passer par le trou. Que d'inventions ne mis-je point en usage pour la tirer! Il fallut trouver (...) A force d'adresse et de temps je parvins à (...) mais à peine furent-elles séparées, qu'elles tombèrent toutes deux dans la dépense. Lecteur pitoyable, partagez mon affliction.

Je ne perdis point courage; mais j'avais perdu beaucoup de temps. Je craignais d'être surpris; je renvoie au lendemain une tentative plus heureuse, et je me remets à l'ouvrage tout aussi tranquillement que si je n'avais rien fait, sans songer aux deux témoins indiscrets qui déposaient contre moi dans la dépense. Le lendemain, retrouvant l'occasion belle, je tente un nouvel essai (....)

L'introducteur un jour, sans être figé, fonctionne comme une sorte de démarcateur épisodique typique du genre narratif et il appelle un indice de clôture du même type, comme le lendemain. Cette attente est au demeurant "leurrée" dans (19) puisque le passage relativement long qui tombe sous la portée de un jour inclut lui-même une allusion au lendemain mais qui n'est pas clôturante (je renvoie au lendemain). Il est possible également que l'alinéa soit interprété dans un premier temps par le lecteur, comme un indice de fermeture du cadre en cours. Quelque hypothèse que l'on adopte à cet égard, il est peu probable que les incises commentatives soulignées dans le texte produisent des hésitations ou rectifications comparables. Les segments sont brefs, marqués syntaxiquement et énonciativement (subjectivisation) et ils spécifient les réactions de Jean-Jacques Rousseau et/ou des lecteurs à un événement particulier rapporté dans la phrase à laquelle ils sont adjoints. Ces ajouts ne tombent pas sous la portée sémantique de un jour, ce qui ne les empêche pas d'être intégrés sous sa portée cadrative. Le fait que ces segments soient clairement évaluatifs et se greffent sur une péripétie évoquée dans une phrase immédiatement précédente, sans compter leur brièveté (il est vrai difficilement prédictible), suggèrent qu'ils ont peu de chances d'être interprétés comme des indices de clôture du cadre en cours. Les cas délicats ne manquent pas et force est de reconnaître qu'il est parfois difficile de décider si un énoncé dont le contenu ne tombe pas clairement sous la portée sémantique d'un introducteur cadratif peut être ou non considéré comme une simple incise, ou si, au contraire, son occurrence est de nature à fermer le cadre en cours (pour une discussion, cf. Sarda, ce volume).

Si l'analyse consistant à faire valoir, comme nous l'avons fait, que l'exploitation à un niveau métadiscursif de composants du contenu propositionnel conduit à considérer qu'ils ne contribuent plus seulement à l'élaboration du sens représentationnel des énoncés, on peut s'attendre à ce que leur interprétation soit sensible à des caractéristiques formelles qui n'ont rien à voir avec les contenus communiqués. Afin d'illustrer ce point nous allons, pour finir, revenir un instant à l'extrait de M.Tournier dont nous sommes partis :

(1)"La vie partagée qu'il menait avait longtemps paru à Edouard un chef-d'œuvre d'organisation heureuse. Aux Pierres Sonnantes il se donnait tout entier aux exigences de l'usine et aux soins de Maria-Barbara et des enfants. À Paris, il redevenait le célibataire oisif et argenté de sa jeunesse.

Pour les besoins de l'analyse, nous avons imaginé une phrase supplémentaire (Il se couchait très tard et dormait peu) et envisagé les effets qui résulteraient de son insertion dans différents endroits du texte lorsque les SP locatifs sont antéposés ou postposés. Dans la discussion du cas (1a) où cette phrase serait rajoutée à la fin de l'extrait original, nous avons fait valoir qu'elle aurait plutôt tendance à être comprise comme tombant dans la portée cadrative du second SP détaché (à Paris), sans toutefois complètement exclure qu'elle puisse être comprise comme faisant allusion au comportement du personnage en général et pas seulement à Paris. Outre la propension "naturelle" des adverbiaux potentiellement cadratifs à étendre leur influence au-delà de leur phrase d'accueil, plusieurs arguments sémantiques 
plaident effectivement en faveur de l'hypothèse d'un rattachement au cadre installé dans la phrase précédente. D'abord il y a le fait que l'on continue à parler d'Edouard (il) et non pas de Maria-Barbara (elle), et ensuite le fait que la phrase rajoutée élabore l'indication qu'il redevenait oisif. Mais ces arguments n'éliminent pas complètement l'hypothèse d'une clôture précoce. Le parallélisme des deux phrases précédentes est tel (SP antéposés, suivis du même pronom sujet, construction avec coordination dans les deux cas) qu'il incite à penser qu'elles constituent un bloc formellement achevé, et que donc la suite (malgré la reprise du pronom et même de la coordination) ne relève pas de la même structure organisationnelle, ce qui favorise le détachement d'avec le cadre en cours.

\section{Conclusion}

Tous les constituants non intégrés syntaxiquement qui sont susceptibles d'apparaître en zone préverbale ne sont pas à même d'initier des cadres de discours et d'assumer, de ce fait, des fonctions organisatrices (Combettes, ce volume). Si l'on ne considère, comme nous l'avons fait dans cet article, que des adverbiaux contribuant au contenu propositionnel des énoncés, il semble que certains sont mieux prédestinés que d'autres à posséder une portée cadrative. Parmi les plus aptes à remplir des fonctions organisatrices, on trouve en première place les spatiaux et les temporels (phrastiques ou non). Ces compléments dits scéniques sont en quelque sorte génériques dans le sens où tout état de choses ayant forcément lieu dans une zone de l'espace et dans un intervalle temporel, il est toujours possible de l'indexer à l'aide de l'une ou l'autre de ces coordonnées (ou des deux ${ }^{18}$. Ce trait explique aussi qu'ils puissent apparaître à l'initiale de discours, sans amorce préalable. D'autres adverbiaux, quoique de sens plus abstrait, constituent également de bons cadratifs. C'est le cas en particulier des praxéologiques (en chimie), ou des médiatifs (selon $X$ ) illustrés dans l'article mais aussi des représentatifs (dans le film/roman de $X$ ) qui sont d'excellents "space builders" (Fauconnier (1984), Huumo (1996, 1999)).

Les compléments précisant les circonstances plus qualitatives des états de choses (comme par hasard) ou les évaluatifs (malheureusement, par chance) ne semblent pratiquement jamais utilisés pour indexer une série de propositions. Les compléments de moyen ou instrumentaux antéposés semblent par contre mieux à même d'assumer des fonctions organisatrices (cf. grâce à ci avant) et cela est encore plus le cas avec les infinitives de but (Charolles \& Lamiroy 2002). Même avec le temps qui est pourtant un bon critère d'indexation, l'opposition est très nette entre les localisateurs qui jouissent d'un large potentiel cadratif et les compléments de durée qui, antéposés, n'exercent en général leur pouvoir cadratif que très localement (Vigier 2004). Il faut aussi tenir compte de la construction de la phrase d'accueil : comme le montrent bien Fuchs \& Fournier (2003), les SP spatiaux ou temporels antéposés dans les phrases à sujet postposé n'ont que des pouvoirs cadratifs très limités.

La liste des SP potentiellement cadratifs est bien entendu ouverte : seules des études sur corpus sont à même de déterminer si tel ou tel adverbial contribuant au contenu propositionnel est susceptible ou non de posséder une portée cadrative, et dans quelles limites.

\section{Références bibliographiques}

BLANCHE-BENVENISTE C., BILGER M., ROUGET C. \& EYNDE K. (1990), Le français parlé : études grammaticales, Paris, CNRS-Editions.

BONAMI, O., GODARD D. \& KAMPERS-MANHE, B. (2004.), "Adverb Classification », in F. Corblin et H. de Swart (eds.), Handbook of French Semantics, Stanford, CSLI Publications.

CHAROLLES M \& LAMIROY B. (2002), "Syntaxe phrastique et transphrastique : du but au résultat", in H.Nolke \& H.L. Andersen eds. Macrosyntaxe et macrosémantique, Bern, Peter Lang, 383-419. 
CHAROLLES M. \& PACHOUD B. (2001), "'Si la Lorpailleur est folle" et si le plus fou n'était pas celle que l'on croit ?", in E.Roulet \& M.Burger eds. Des modèles du discours au défi d'un "dialogue romanesque". L'incipit du roman de R.Pinget "Le libera"", Nancy, PUN,307-350.

CHAROLLES M. (1997), L'encadrement du discours : univers, champs, domaines et espaces, Cahier de Recherche Linguistique, LANDISCO, URA-CNRS 1035 Université Nancy 2, $\mathrm{n}^{\circ}$ 6, 1-73. Accessible sur le site http://www.lattice.cnrs.fr/.

CHAROLLES M. (2003), "De la topicalité des adverbiaux détachés en tête de phrase", Travaux de Linguistique, 47, 11-51.

CHAROLLES M., (à par.), "De la cohérence à la cohésion", actes du colloque Cohérence et Discours, Tunis, 16-20/02/2005, Presses Universitaires de la Sorbonne.

CHAROLLES M., LE DRAOULEC A., PERY-WOODLEY M.P. \& SARDA L. (à par.), "Temporal and spatial dimensions of discourse organisation", Journal of French Language Studies.

COMBETTES B. (2003), " Le topique comme constituant périphérique : aspects diachroniques", Travaux de Linguistique, 47, 137-162.

DANELL K. J. (1998), "La portée comme phénomène linguistique", Le Français moderne, 66 (1), 1-26.

GUIMIER C. (1996), Les adverbes du français. Le cas des adverbes en -ment, Paris, Ophrys.

FAUCONNIER G. (1984), Espaces mentaux, Paris, Minuit

FUCHS C. \& FOURNIER N. (2003), "Du rôle cadratif des compléments localisants initiaux selon la position du sujet nominal", Travaux de Linguistique, 47, 79-110.

HALLIDAY M.A.K. \& HASAN R. (1976), Cohesion in English, London, Longman.

HUUMO T. (1996), "A scoping hierarchy of locatives", Cognitive linguistics, 7-3, 265-299.

HUUMO T. (1999), "Space and time : temporalization and other special functions of locatting-setting", Linguistics, 37-3, 389-430.

LACHERET-DUJOUR A \& VICTORRI B. (2002), "La période intonative comme unité d'analyse pour l'étude du français parlé : Modélisation prosodique et enjeux linguistiques", Verbum, XXIV, 1-2, 55-72.

LACHERET-DUJOUR A, PLOUX \& VICTORRI B. (1998), "Prosodie et thématisation en français parlé", in C.Fuchs \& Ch.Marchello-Nizia eds, Cahiers de Praxématique, 30, 89-111.

LAHOUSSE K. (2003), "La complexité de la notion de topique et l'inversion du sujet nominal", Travaux de Linguistique, 47, 111-136.

LE DRAOULEC, A., \& PÉRY-WOODLEY, M.-P. (2003), "Time travel in text: temporal framing in narratives and nonnarratives", in L. Lagerwerf, W. Spooren, \& L. Degand (Eds.), Determination of Information and Tenor in Texts: Multidisciplinary Approaches to Discourse, Amsterdam/Münster: Stichting Neerlandistiek \& Nodus Publikationen, 267-275.

MOLINIER C. \& LEVRIER F. (2000), Grammaire des adverbes. Description des formes en -ment, Genève, Droz.

MOREL M.A. \& DANON-BOILEAU L (1998), Grammaire de l'intonation. L'exemple du français, Paris, FDL, Ophrys.

MULLER C. (1991), La négation en français, Genève, Droz.

MULLER C. (2005), "Portée et phrases négatives à modifieurs adverbiaux", in I.Choi-Jonin, M.Bras, A.Dagnac \& M.Rouquier eds., Questions de classification en linguistique : méthodes et descriptions, Berne, Peter Lang, 241-262.

NEVEU F. (2000), Lexique des notions linguistiques, Paris, Nathan-Université.

NØLKE H. (1993), Le regard du locuteur. Pour une linguistique des traces énonciatives, T1, Paris, Kimé.

NØLKE H. (1994), Linguistique modulaire : de la forme au sens, Louvain-Paris, Peeters.

NØLKE H. (2001), Le regard du locuteur. Pour une linguistique des traces énonciatives, T2, Paris, Kimé.

PREVOST S. (2003), 'Les compléments spatiaux : du topique au focus en passant par les cadres", Travaux de Linguistique, 47, 51-78.

RIEGEL M., PELLAT J.C. \& RIOUL R. (1994), Grammaire Méthodique du Français, Paris, PUF.

RIVENC, F. (1989), Introduction à la logique, Paris, Payot.

ROSSARI C. (1997), Les opérations de reformulation. Analyse du processus et des marques dans une perspective contrastive français-italien, $2^{\mathrm{e}}$ éd.,Bern, Peter Lang.

SCHREPFER-ANDRE G. (2004), "Sur la portée textuelle des expressions introductrices de cadres de discours en selon X : les indices de clôture des univers énonciatifs", in Delamotte-Legrand, R. et al. (eds), Les médiations langagières, vol. 1, Des faits de langue aux discours, Actes du colloque international La médiation : marquages en langue et en discours, Dyalang CNRS, Publications de l'Université de Rouen.

TERRAN, E. (2002), Le cadrage temporel en français, Thèse de Doctorat, Université de Paris III.

THOMPSON S. (1985), "Grammar and written discourse : Initial vs. Final purpose clauses in English", Text, 5, 1-2, 55-84.

VIGIER D. (2003), "Les syntagmes prépositionnels en «en $\mathrm{N}$ » détachés en tête de phrase référant à des domaines d'activité", Lingvisticae Investigationes, 26 (1), 97 - 122, Amsterdam, John Benjamins B.V.

VIGIER, D. (2004), Les groupes prépositionnels en « en N » : de la phrase au discours, Thèse de Doctorat, Université de Paris III. 